\title{
Article \\ Simultaneous Enhancement of Contrast and Power of Femtosecond Laser Pulses by Nonlinear Interferometer
}

\author{
Yasser Nada ${ }^{1, *}$ and Efim Khazanov ${ }^{2}$ \\ 1 Physics Department, Faculty of Science, Menofia University, Shibin El-Kom, Menofia 32511, Egypt \\ 2 Institute of Applied Physics, Russian Academy of Sciences, Nizhny Novgorod 603950, Russia; \\ efimkhazanov@gmail.com \\ * Correspondence: yasersci@hotmail.com
}

Citation: Nada, Y.; Khazanov, E. Simultaneous Enhancement of Contrast and Power of Femtosecond Laser Pulses by Nonlinear Interferometer. Photonics 2021, 8, 520. https://doi.org/10.3390/ photonics 8110520

Received: 27 October 2021

Accepted: 17 November 2021

Published: 19 November 2021

Publisher's Note: MDPI stays neutral with regard to jurisdictional claims in published maps and institutional affiliations.

Copyright: (c) 2021 by the authors. Licensee MDPI, Basel, Switzerland. This article is an open access article distributed under the terms and conditions of the Creative Commons Attribution (CC BY) license (https:/ / creativecommons.org/licenses/by/ $4.0 /)$.
Abstract: We showed that the nonlinear Mach-Zehnder interferometer may be used not only for enhancing temporal contrast, as proposed earlier, but also for increasing pulse power due to efficient pulse compression. The interferometer introduces into the output pulse a nonlinear phase equal to $\pi / 2$. This allows increasing laser power by a factor of 1.5 only by means of a chirped mirror. Use of an additional nonlinear plate leads to a multi-fold power increase retaining the contrast enhancement.

Keywords: nonlinear Mach-Zehnder interferometer; post compression; femtosecond laser pulse contrast; self phase modulation

\section{Introduction}

High temporal contrast has an important role in experiments on studying the behavior of matter in extreme light fields. Temporal contrast is the ratio of the intensity at the peak of the pulse to the intensity on its pedestal. The pedestal appears, as a rule, due to amplified spontaneous emission in laser amplifiers of CPA lasers (chirped pulse amplification) [1], or due to amplified parametric emission in OPCPA lasers (optical parametric chirped pulse amplification) [2]. The most popular techniques for contrast enhancement are plasma mirrors [3], harmonic generation [4], and cross-polarized wave (XPW) generation [5]. XPW is based on cubic nonlinearity. Recently, several new ideas have been proposed for contrast enhancement, by means of a nonlinear phase induced by cubic nonlinearity: spectral filtering [6,7], spatial filtering [8], a nonlinear polarization interferometer [9], as well as a nonlinear Mach-Zehnder interferometer with symmetric arms [10]. In the latter case, the phase difference without non-linearity $\Delta \varphi_{\mathrm{L}}$ equals $\pi$, and the radiation of the pedestal does not pass to the dark port (see Figure 1a). On the contrary, the main pulse acquires a nonlinear phase B (B-integral) in one channel, and a zero nonlinear phase in another one. If $\mathrm{B}$ is nonzero, then the dark port does not become completely dark. If $\Delta \mathrm{B}=\pi$, then the dark port becomes a light port, and the major part of the main pulse comes through this port. As a result, the contrast will be infinitely high if $\Delta \varphi=\pi$. In practice, the contrast enhancement is determined by the inaccuracy of meeting the condition $\Delta \varphi=\pi$.

Due to self-phase modulation (SPM) in a nonlinear medium, and subsequent reflection from the chirped mirror (CM), the pulse may be compressed, and hence the peak power increases. The method is called TFC (thin film compression) [11], CafCA (compression after compressor approach) [12], or post-compression [13]. For pulses with energy of tens of Joules [14-16], the multiple compression of femtosecond laser pulses was demonstrated with almost no energy loss; for more details, see the review [17].

In this paper, we show that a nonlinear Mach-Zehnder interferometer may be used not only for contrast enhancement, but also for multi-fold pulse compression simultaneously. 


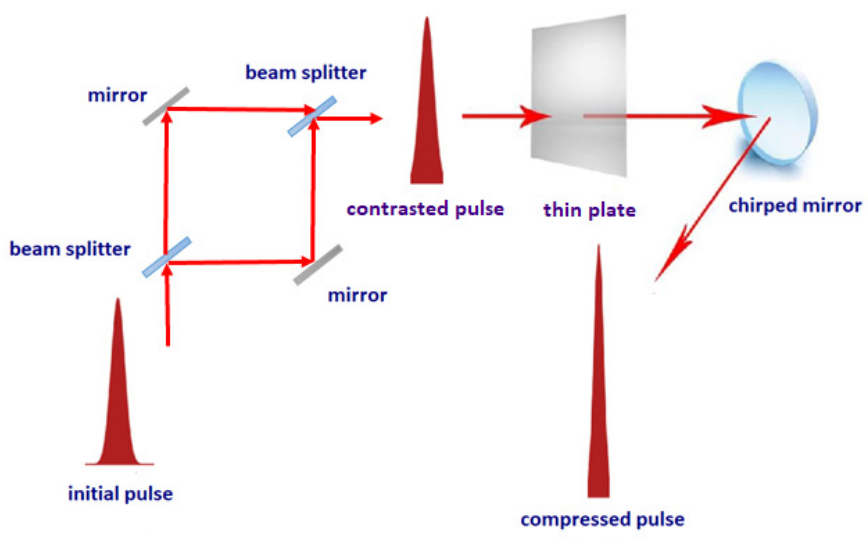

( a )

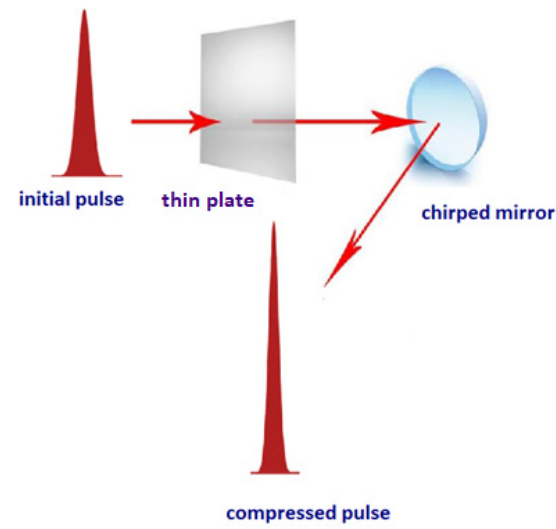

(b)

Figure 1. Optical schemes with nonlinear Mach-Zehnder interferometer (a) and without interferometer (b).

\section{Nonlinear Mach-Zehnder Interferometer for Enhancement of Contrast and Pulse Compression}

For the Mach-Zehnder interferometer (see Figure 1a), the expressions for intensities $\mathrm{I}_{1}$ and $I_{2}$ at the outputs of the arms (ports) have the form [10]

$$
\begin{gathered}
\mathrm{I}_{1}(\mathrm{t})=\{1-2(1-\mathrm{R}) \mathrm{R}+2(1-\mathrm{R}) \mathrm{R} \cos [\Delta \varphi+2(1-\mathrm{R}) \mathrm{B}(\mathrm{t})]\} \mathrm{I}_{\mathrm{o}}(\mathrm{t}) . \\
\mathrm{I}_{2}(\mathrm{t})=\{2(1-\mathrm{R}) \mathrm{R}+2(1-\mathrm{R}) \mathrm{R} \cos [\Delta \varphi+2(1-\mathrm{R}) \mathrm{B}(\mathrm{t})]\} \mathrm{I}_{\mathrm{o}}(\mathrm{t}) .
\end{gathered}
$$

Here, $\mathrm{I}_{0}$ is the intensity at the interferometer input $\left(\mathrm{I}_{0}=\mathrm{I}_{1}+\mathrm{I}_{2}\right) ; \Delta \varphi$ is the linear phase difference acquired by the pulses during propagation along the interferometer arms; $\mathrm{B}(\mathrm{t})=(2 \pi / \lambda) \mathrm{I}_{\mathrm{o}}(\mathrm{t}) n_{2} L$ is the nonlinear phase (B-integral) accumulated in both beam splitters; $L$ is length of the beam path in the beam splitters; $\lambda$ is the wavelength; $n_{2}$ is the nonlinear refractive index; and $R$ is the reflectivity of the beam splitters.

Under the conditions $\Delta \varphi=\pi$ and $\mathrm{R}=0.5$, the value of $\mathrm{I}_{1}$ in Equation (1) may be exactly zero in the absence of a nonlinear phase $(B=0)$. On the other hand, at high intensity, the nonlinear phase is accumulated, and the intensity $\mathrm{I}_{1}$ takes on a maximal value, provided that $\mathrm{B}=\pi, \Delta \varphi=\pi$ and $\mathrm{R}=0.5$. As a result, the pulse emerging at this port has a higher contrast. Moreover, the pulse duration shortened after reflection from the CM. For example, an input pulse of $50 \mathrm{fs}$ may be shortened to $38 \mathrm{fs}$ [10], which is a tiny compression factor.

The first way to increase the compression factor is to use a CM to remove the chirp from the pulse. It is easy to show that the output pulse $\mathrm{I}_{1}$ accumulates a nonlinear phase $\mathrm{B}_{\text {out }}=\pi / 2$ because output pulse accumulates nonlinear phase $\mathrm{B}_{\text {out }}=(\mathrm{B} 1+\mathrm{B} 2) / 2$. Where, $\mathrm{B} 1$ and $\mathrm{B} 2$ is nonlinear phases in the interferometer arms 1 and 2 , respectively. Since $\mathrm{B} 1=\pi$ and $B 2=0$, therefore $B_{\text {out }}=\pi / 2$. As shown below, this acquired nonlinear phase leads to compression to about $25 \mathrm{fs}$, which is still a modest value, because of a small value of $B_{\text {out }}$. The second way is to introduce an additional nonlinear phase $B_{\text {add }}$ by adding an additional nonlinear plate before the CM (see Figure 1a). Thus, the total value of the B-integral is $\mathrm{B}_{\Sigma}=\pi / 2+\mathrm{B}_{\text {add }}$. We will study the efficiency of pulse compression in this case. As a reference, we will use compression without interferometer (without contrast enhancement) with the B-integral equal to $B_{\Sigma}$ (Figure $1 b$ ). The reference case is obviously more robust and practical, but it does not provide any impact on the pedestal.

Contrast enhancement is purely linear physics, but pulse compression is nonlinear, so numerical calculation is needed. We performed a detailed numerical study and comparison of compression efficiency with (Figure 1a) and without (Figure 1b) interferometer.

\section{Numerical Model}

Pulse propagation in a nonlinear plate is described by the nonlinear Schrödinger Equation (3) [18]

$$
i \frac{\partial A}{\partial \ddagger}-\alpha \frac{\partial^{2} A}{\partial \tau^{2}}+\beta|A|^{2} A+i \gamma \frac{\partial}{\partial \tau}\left(|A|^{2} A\right)=0
$$


where $A$ is the normalized amplitude of the E-field, $\tau$ is the normalized time, and the coefficients $\alpha, \beta$ and $\gamma$ are defined as

$$
\alpha=\frac{1}{2} \frac{\partial^{2} k}{\partial \omega^{2}}\left(\omega_{0}\right), \beta=\frac{n_{2} \omega_{0}}{c}, \gamma=\frac{2 n_{2}}{c}
$$

where $\omega_{0}$ is the central frequency of the pulse.

We used Equation (3) for the numerical modeling of pulse propagation, both in the interferometer's beam splitters, and in thin plates. Equation (3) is solved numerically by the split step Fourier method (SSFM). This method is widely used for solving the problem of pulse propagation in a nonlinear dispersive medium. The main advantage of this method is the use of the fast Fourier transform (FFT) algorithm, which makes it faster than the finite difference methods. Therefore, we have developed a MATLAB algorithm to implement the numerical FFT algorithm. To demonstrate the compression of the pulse as a function of $B_{\Sigma}$, we scanned the thickness of the nonlinear media, keeping the other parameters constant: input intensity $1 \mathrm{TW} / \mathrm{cm}^{2}$, input pulse duration (FWHM) $50 \mathrm{fs}$ and $30 \mathrm{fs}$, and wavelength $910 \mathrm{~nm}$. We chose material parameters of fused silica as the most popular material: nonlinear refractive index $n_{2}=2.75 \times 10^{-16} \mathrm{~cm}^{2} / \mathrm{W}$ and group velocity dispersion $\alpha=280 \mathrm{fs}^{2} / \mathrm{cm}$.

SPM leads to spectral broadening; the pulse becomes positively chirped. Thus, the pulse may be compressed by reflection on the $\mathrm{CM}$ with negative dispersion (Figure 1). We restrict the consideration to the case in which the $\mathrm{CM}$ introduced a purely quadratic spectral phase, i.e., group velocity dispersion only. Such a type of CMs cannot compress the pulse to a Fourier transform limit, but they are usually commercially available. In this case, the CM is embedded in the model using Equation (4)

$$
A_{\text {compressed }}(t)=\mathbf{F}\left[e^{\frac{-i \alpha\left(\omega-\omega_{0}\right)^{2}}{2}} \mathbf{F}^{-1}\left(A_{\text {chirped }}(t, z)\right)\right]
$$

where $A_{\text {compressed }}$ is the amplitude after compression (after reflection from the $\mathrm{CM}$ ) and $A_{\text {chirped }}$ is the amplitude of the field incident on the CM, F and $\mathbf{F}^{-1}$ are the direct and inverse Fourier transforms, respectively. The parameter $\alpha$ is the group velocity dispersion parameter of the CM.

Using Equations (3) and (4), we found the output pulses both for the setup with (Figure 1a) and without interferometer (Figure 1b).

\section{Results and Discussion}

The primary effect of SPM is spectral broadening. So, first of all, we compare the spectral bandwidth before the CM. Then, we should choose the CM dispersion $\alpha_{\text {opt }}$. It may be chosen to minimize the compressed pulse duration or to maximize peak power. However, we prefer the last case, because increasing the pulse power is a primary goal for most applications. Furthermore, we study pulse shortening and power enhancement.

\subsection{Spectral Broadening}

The results of calculations are shown in Figure 2. The pulse in the scheme with interferometer has a wider spectral bandwidth than that without interferometer, even though $B_{\Sigma}$ is the same. This phenomenon is explained as follows. At the interferometer output (Figure 1a), the beam is a sum of two beams: one with $B=0$ and the other with $B=\pi$. The spectrum of the first beam is not broadened at all, while the spectrum of the second one is broadened much stronger than the spectrum of the single beam with $B_{\Sigma}=\pi / 2$ in the reference case (Figure 1b). In other words, due to the nonlinear nature of SPM, the spectral broadening with interferometer is higher than in the case without the interferometer, even if $\mathrm{B}_{\Sigma}=\pi / 2$ in both cases (see Figure $2 \mathrm{a}, \mathrm{c}$ ). An additional nonlinear plate increases $\mathrm{B}_{\Sigma}$ up to 5 , but keeps this difference (Figure 2b,d). 


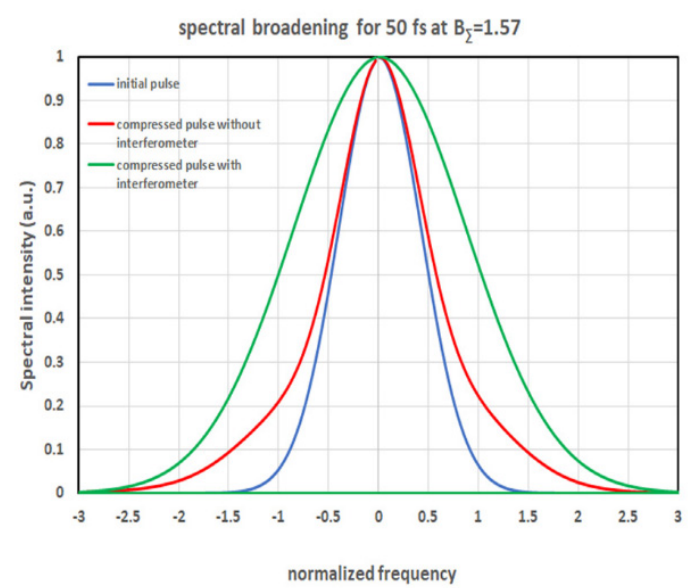

a

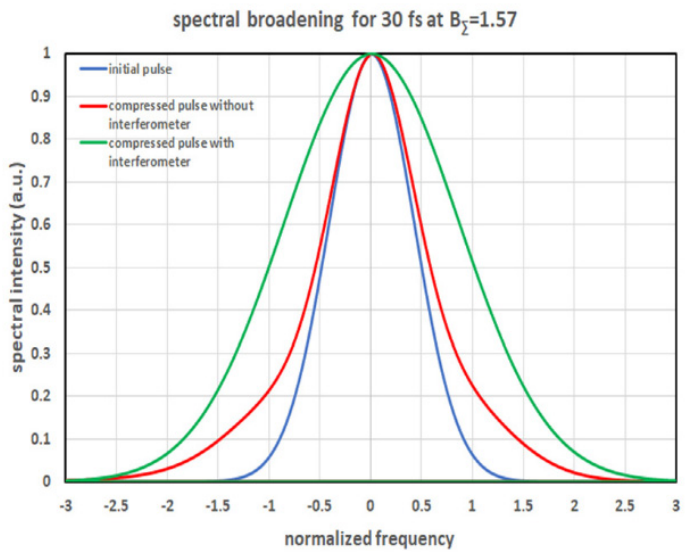

c

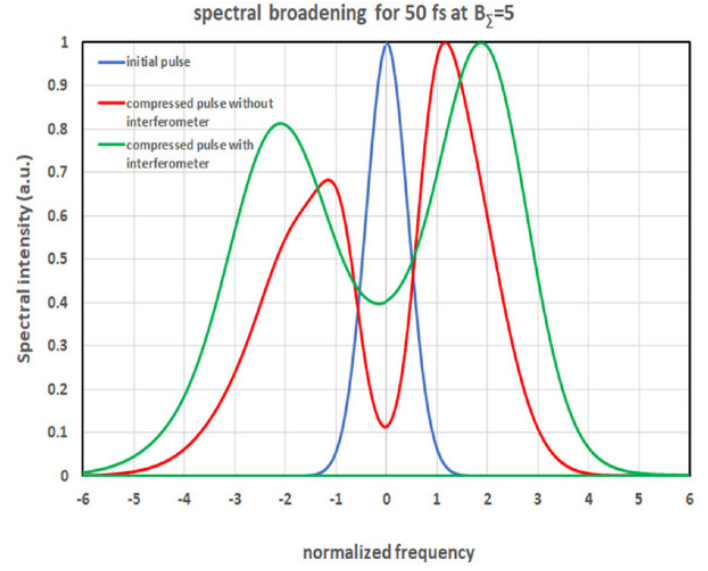

b

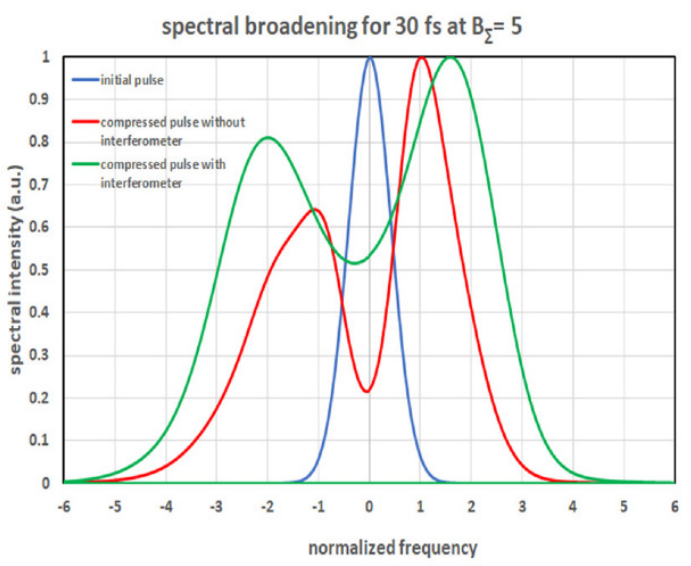

d

Figure 2. Spectrum of the initial pulse, compressed pulse in the scheme with interferometer (Figure 1a), and compressed pulse in the scheme without interferometer (Figure $1 \mathrm{~b}$ ) for $50 \mathrm{fs}(\mathbf{a}, \mathbf{b})$ and $30 \mathrm{fs}(\mathbf{c}, \mathbf{d})$ input pulses at $B_{\Sigma}=\pi / 2(\mathbf{a}, \mathbf{c})$ and $B_{\Sigma}=5$ $(\mathbf{b}, \mathbf{d})$. Horizontal axes are normalized to the input pulse bandwidth.

The spectra for $50 \mathrm{fs}$ and $30 \mathrm{fs}$ input pulses are very similar (note that the horizontal axes are normalized to the input pulse bandwidths $8.82 \times 10^{12} \mathrm{~Hz}$ and $1.47 \times 10^{13} \mathrm{~Hz}$ for $50 \mathrm{fs}$ and $30 \mathrm{fs}$, respectively). The small difference between $50 \mathrm{fs}$ and $30 \mathrm{fs}$ at $B_{\Sigma}=5$ is due to the fact that the bandwidth for $30 \mathrm{fs}$ input pulse becomes comparable to the optical frequency.

\subsection{Optimal Chirped Mirror Dispersion $\alpha_{\text {opt }}$}

As mentioned above, we defined the optimal CM dispersion $\alpha_{\mathrm{opt}}$ as a dispersion which maximizes compressed pulse peak power. When $\mathrm{CM}$ dispersion is not exactly equal to $\alpha_{\mathrm{opt}}$, the compressed pulse power is smaller. We calculated $\alpha_{\mathrm{opt}}$ as a function of $\mathrm{B}_{\Sigma}$. The results are shown in Figure 3, from which it is seen that correcting the quadratic spectral phase component for higher values of the B-integral necessitates a lower (in modulus) chirped mirror dispersion $\alpha_{\mathrm{opt}}$. Moreover, it is clear from Figure 3 that $\alpha_{\mathrm{opt}}$ is smaller in the case with interferometer than without it. The smaller value of $\alpha_{\text {opt }}$ provides a practical advantage, because the fabrication of $\mathrm{CM}$ with a higher dispersion is a more serious challenge. It is worth noticing that, at a high value of B-integral, $\alpha_{\text {opt }}$ is almost independent of $B_{\Sigma}$. Hence, the same CM may be used for a B-integral in a wide range of values, eliminating the impact of instability of the laser parameters. 


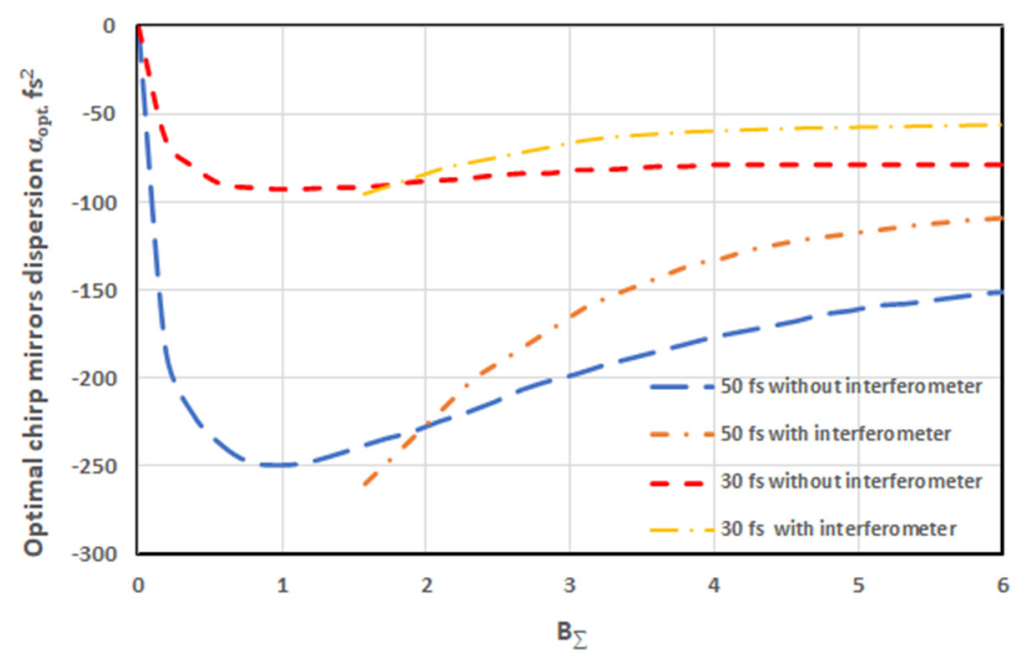

Figure 3. $B_{\Sigma}$-dependence of parameter $\alpha_{\mathrm{opt}}$ for different pulse durations with and without interferometer.

\subsection{Pulse Compression}

The results are shown in Figure 4. The compressed pulse in the scheme with interferometer is even shorter than in the scheme without interferometer. It is explained by the wider spectrum (see Figure 2). For instance, at $B_{\Sigma}=5$, for $50 \mathrm{fs}$ input pulse, the compressed pulse duration decreased to about $14 \mathrm{fs}$ in the scheme without interferometer, and to about $12 \mathrm{fs}$ with interferometer; for the $30 \mathrm{fs}$ input pulse, the compressed pulse duration decreased to about $9 \mathrm{fs}$ in the case without interferometer, and to about $7 \mathrm{fs}$ in the scheme with interferometer.

In addition, the intensity in the compressed pulse wings is lower in the case with interferometer because the interferometer remains closed for the input pulse tails, and the chirp in the tails differs greatly from the linear chirp. So, removing the tails from the input pulse causes the compressed pulse to be closer to the Fourier transform limited one (cf. the green and red curves in Figure 4). Thus, from the pulse compression viewpoint, the case with interferometer (Figure 1a) is more preferable than the reference case (Figure 1b).

\subsection{Peak Power Increase}

From the viewpoint of peak power, the case with interferometer (Figure 1a) strongly differs from the reference case (Figure 1b). The latter is energy lossless, while the first one is not. Energy is lost because the dark port of the interferometer becomes perfectly light only at $\mathrm{B}=\pi$, i.e., only at $t=0$, i.e., for the central part of the pulse. For $t \neq 0$, the interferometer transmission is below $100 \%$ by virtue of $B \neq \pi$. For the pulse periphery, $\mathrm{B}<<\pi$ and the pulse do not pass through the interferometer at all. The energy transmission of the interferometer for a Gaussian pulse with $\mathrm{B}(t=0)=\pi$ is $76 \%$ for any pulse duration. This inevitable disadvantage reduces the power of compressed pulses. Nevertheless, as seen from Figure 4, the peak power is almost the same for both cases. Figure 5 shows that this is true for any value of B-integral. In spite of $24 \%$ energy loss in the interferometer, the superiority of the case without interferometer is below $10 \%$. This is explained by more efficient pulse compression in the case with the interferometer. 


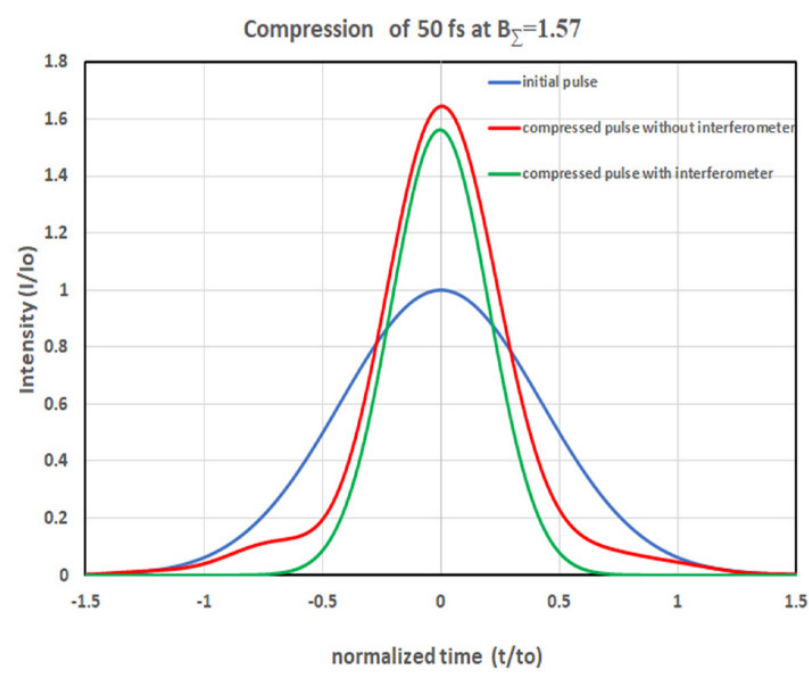

a

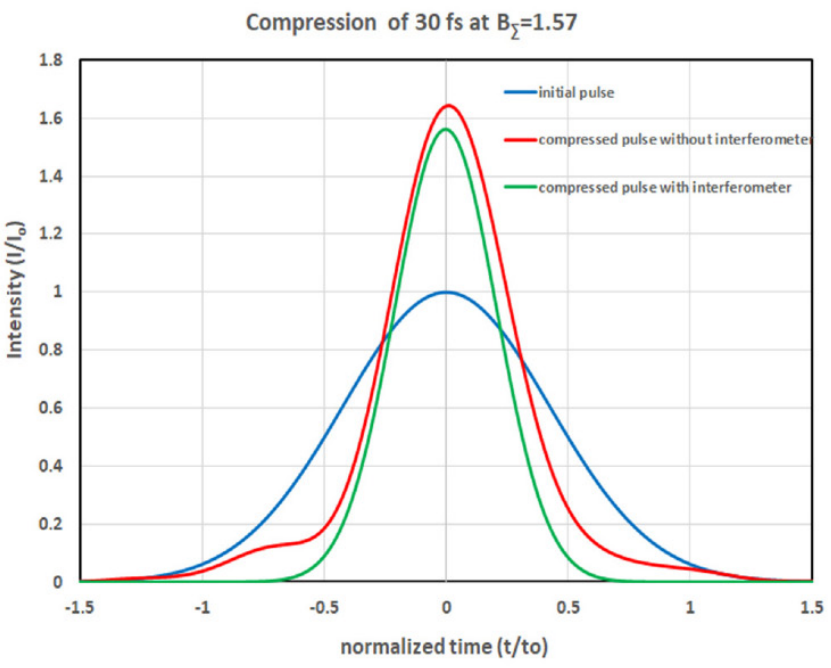

C

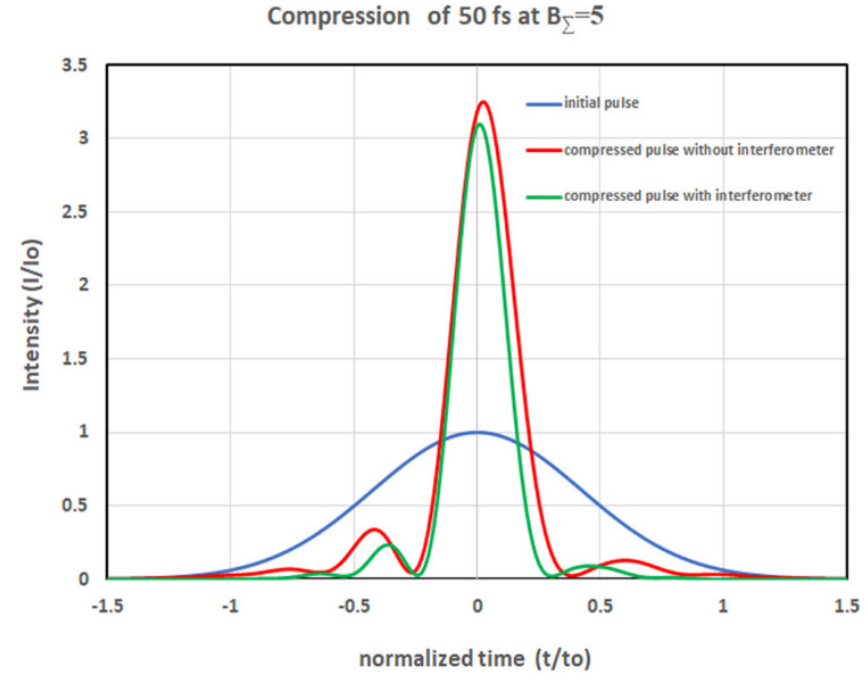

b

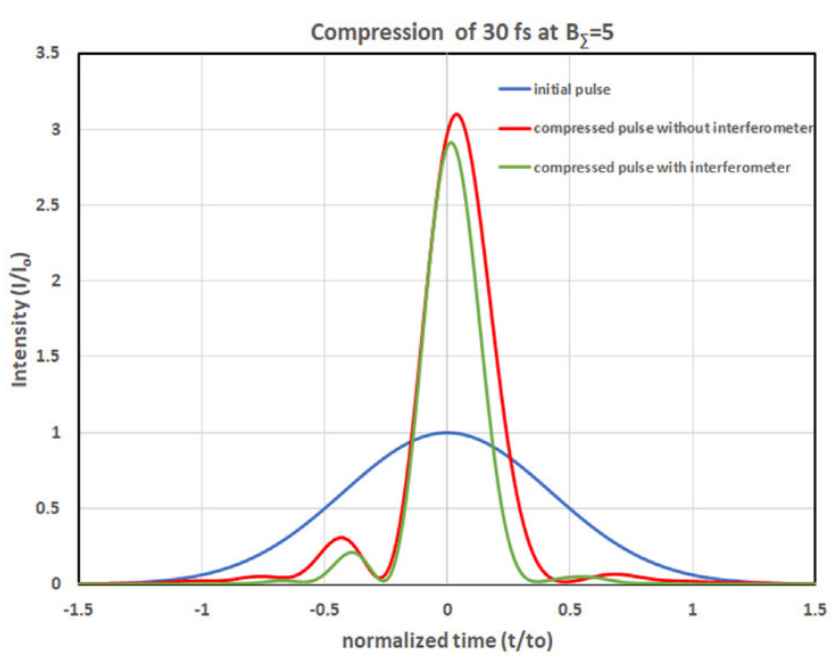

d

Figure 4. Shapes of the initial pulse, compressed pulse in the scheme with interferometer (Figure 1a) and compressed pulse in the scheme without interferometer (Figure $1 \mathrm{~b})$ for $50 \mathrm{fs}(\mathbf{a}, \mathbf{b})$ and $30 \mathrm{fs}(\mathbf{c}, \mathbf{d})$ input pulses at $B_{\Sigma}=\pi / 2(\mathbf{a}, \mathbf{c})$ and $B_{\Sigma}=5(\mathbf{b}, \mathbf{d})$.

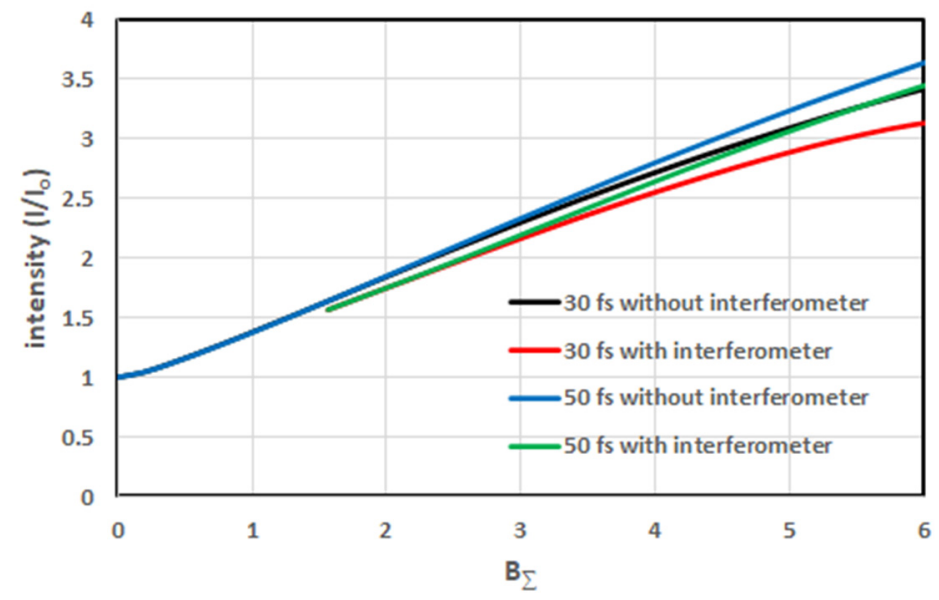

Figure 5. Output pulse intensity as a function of B- integral. 


\section{Conclusions}

Numerical modeling confirmed that the nonlinear Mach-Zehnder interferometer may be used not only to enhance the temporal contrast, but also to increase the pulse power due to efficient pulse compression. The pulse shortens due to self-phase modulation and a chirped mirror. Self-phase modulation occurs during propagation, both in the interferometer beam splitters, and in an additional nonlinear plate. We showed that pulse compression in the scheme with interferometer is either the same or even better than the standard compression in the scheme without interferometer and without contrast improvement.

Author Contributions: Conceptualization, E.K.; methodology, Y.N.; software, Y.N.; validation, E.K. and Y.N.; formal analysis E.K.; investigation, Y.N.; resources, Y.N.; data curation, Y.N.; writingoriginal draft preparation, E.K. and Y.N.; writing-review and editing, E.K. and Y.N.; visualization, E.K.; supervision E.K.; project administration, E.K.; funding acquisition, E.K.; All authors have read and agreed to the published version of the manuscript.

Funding: This research was supported by the Center of Excellence "Center of Photonics" funded by the Ministry of Science and Higher Education of the Russian Federation, agreement No. 075-15-2020-906.

Institutional Review Board Statement: Not applicable.

Informed Consent Statement: Not applicable.

Data Availability Statement: Not applicable.

Acknowledgments: This work was supported by the Center of Excellence "Center of Photonics" funded by the Ministry of Science and Higher Education of the Russian Federation, agreement No. 075-15-2020-906.

Conflicts of Interest: The authors declare no conflict of interest.

\section{References}

1. Strickland, D.; Mourou, G. Compression of amplified chirped optical pulses. Opt. Commun. 1985, 56, 219-221. [CrossRef]

2. Dubietis, A.; Jonusauskas, G.; Piskarskas, A. Powerful femtosecond pulse generation by chirped and stretched pulse parametric amplification in BBO crystal. Opt. Commun. 1992, 88, 437-440. [CrossRef]

3. Lévy, A.; Ceccotti, T.; D’Oliveira, P.; Réau, F.; Perdrix, M.; Quéré, F.; Monot, P.; Bougeard, M.; Lagadec, H.; Martin, P.; et al. Double plasma mirror for ultrahigh temporal contrast ultraintense laser pulses. Opt. Lett. 2007, 32, 310-312. [CrossRef] [PubMed]

4. Mironov, S.Y.; Lozhkarev, V.V.; Ginzburg, V.N.; Khazanov, E.A. High-efficiency second-harmonic generation of superintense ultrashort laser pulses. Appl. Opt. 2009, 48, 2051-2057. [CrossRef] [PubMed]

5. Jullien, A.; Albert, O.; Burgy, F.; Hamoniaux, G.; Rousseau, J.-P.; Chambaret, J.-P.; Augé-Rochereau, F.; Chériaux, G.; Etchepare, J.; Minkovski, N.; et al. 10^-10 temporal contrast for femtosecond ultraintense lasers by cross-polarized wave generation. Opt. Lett. 2005, 30, 920-922. [CrossRef] [PubMed]

6. Buldt, J.; Müller, M.; Klas, R.; Eidam, T.; Limpert, J.; Tünnermann, A. Temporal contrast enhancement of energetic laser pulses by filtered self-phase-modulation-broadened spectra. Opt. Lett. 2017, 42, 3761-3764. [CrossRef] [PubMed]

7. Mironov, S.Y.; Starodubtsev, M.V.; Khazanov, E.A. Temporal contrast enhancement and compression of output pulses of ultra-high power lasers. Opt. Lett. 2021, 46, 1620-1623. [CrossRef] [PubMed]

8. Khazanov, E.A. Enhancing the time contrast and power of femtosecond laser pulses by an optical wedge with cubic nonlinearity. Quantum Electron. 2021, 51, 433-436. [CrossRef]

9. Khazanov, E. Nonlinear polarization interferometer for enhancement of laser pulse contrast and power. Opt. Express 2021, 29, 17277-17285. [CrossRef] [PubMed]

10. Khazanov, E.A.; Mironov, S.Y. Nonlinear interferometer for increasing the contrast ratio of intense laser pulses. Quantum Electron. 2019, 49, 337-343. [CrossRef]

11. Mourou, G.; Mironov, S.; Khazanov, E.; Sergeev, A. Single cycle thin film compressor opening the door to Zeptosecond-Exawatt physics. Eur. Phys. J. Spec. Top. 2014, 223, 1181-1188. [CrossRef]

12. Ginzburg, V.N.; Yakovlev, I.V.; Zuev, A.S.; Korobeinikova, A.A.; Kochetkov, A.A.; Kuz'min, A.A.; Mironov, S.Y.; Shaykin, A.A.; Shaykin, I.A.; Khazanov, A.E. Compression after compressor: Threefold shortening of 200-TW laser pulses. Quantum Electron. 2019, 49, 299-301. [CrossRef]

13. Balla, P.; Bin Wahid, A.; Sytcevich, I.; Guo, C.; Viotti, A.-L.; Silletti, L.; Cartella, A.; Alisauskas, S.; Tavakol, H.; Grosse-Wortmann, U.; et al. Postcompression of picosecond pulses into the few-cycle regime. Opt. Lett. 2020, 45, 2572-2575. [CrossRef] [PubMed]

14. Ginzburg, V.; Yakovlev, I.; Zuev, A.; Korobeynikova, A.; Kochetkov, A.; Kuzmin, A.; Mironov, S.; Shaykin, A.; Shaikin, I.; Khazanov, E.; et al. Fivefold compression of 250-TW laser pulses. Phys. Rev. A 2020, 101, 013829. [CrossRef] 
15. Ginzburg, V.N.; Yakovlev, I.V.; Zuev, A.S.; Korobeynikova, A.P.; Kochetkov, A.A.; Kuzmin, A.A.; Mironov, S.Y.; Shaykin, A.A.; Shaikin, I.A.; Khazanov, E.A. Two-stage nonlinear compression of high-power femtosecond laser pulses. Quantum Electron. 2020, 50, 331-334. [CrossRef]

16. Ginzburg, V.; Yakovlev, I.; Kochetkov, A.; Kuzmin, A.; Mironov, S.; Shaikin, I.; Shaykin, A.; Khazanov, E. 11 fs, 1.5 PW laser with nonlinear pulse compression. Opt. Express 2021, 29, 28297-28306. [CrossRef] [PubMed]

17. Khazanov, E.A.; Mironov, S.Y.; Mourou, G. Nonlinear compression of high-power laser pulses: Compression after compressor approach. Phys.-Uspekhi 2019, 62, 1096-1124. [CrossRef]

18. Anderson, D.P.; Lisak, M. Nonlinear asymmetric self-phase modulation and self-steepening of pulses in long optical waveguides. Phys. Rev. A 1983, 27, 1393-1398. [CrossRef] 